\title{
TENDENCIAS DE LA INVESTIGACIÓN EN ENFERMERÍA
}

\author{
Elizabeth Murrain Knudson*
}

\section{Resumen}

El contexto actual del desarrollo académico y administrativo de las facultades y programas universitarios representa una oportunidad de autoevaluación y para formular planes de mejoramiento continuo a través de procesos como el de requisitos mínimos de funcionamiento (registro calificado), la acreditación nacional e internacional y la reacreditación tanto nacional como internacional. Más allá de la obtención de la certificación correspondiente por parte del Ministerio de Educación Nacional, promueve en la comunidad académica la cultura de autoevaluación con fines de mejoramiento continuo y aquello que hace décadas constituía un "desgaste tormentoso", hoy se convierte en un estilo de vida para las instituciones universitarias, puesto que mirar, evaluar, transformar, mejorar y trascender hace parte del crecimiento y desarrollo de la comunidad que conforma cada institución de educación superior. Esa por lo menos ha sido la mirada establecida por la Fundación Universitaria de Ciencias de la Salud. En el presente artículo se observa el camino que ha recorrido la Facultad de Enfermería en el desarrollo disciplinar, social, político y económico de la salud colombiana, a través de las huellas generadas con los proyectos de investigación de los estudiantes que han cursado las especializaciones tanto clínicas como interdisciplinarias. Consideramos importante presentar a la comunidad académica la esencia de los pasos y caminos recorridos, teniendo en cuenta que la Facultad de Enfermería de la Fundación constituye una de las doce mejores del país, lo cual se respalda en tres importantes procesos: uno, en la acreditación otorgada por el Ministerio de Educación Nacional; el segundo, en la posición que ocupa al estar entre las diez primeras del país debido a los resultados obtenidos por nuestros estudiantes en los Ecaes desde la creación de éstos hace ya cinco años; y el tercero, el posicionamiento y reconocimiento por parte de los empleadores de entidades públicas y privadas, exaltando la claridad y calidad profesional tanto humana como técnica y científica, sello de garantía disciplinar e institucional. Se realizó un análisis de las diferentes tesis presentadas por los egresados desde 1997 hasta el primer semestre de 2008, en cuanto a nombre, tema abordado, área del conocimiento y metodologías utilizadas.

Palabras clave: tendencias, investigación.

\section{TRENDS IN NURSING RESEARCH}

\section{Abstract}

The current context of administrative and academic development of the schools and university programs provides an opportunity to conduct critical introspection as well as to propose continuous improvement plans through processes such as the achievement of minimum quality requirements (quality records), and both national and international accreditation and re-accreditation. Far more than obtaining the corresponding National Ministry of Education certification it encourages

Fecha recibido: julio 17 de 2008 - Fecha aceptado: agosto 4 de 2008

* Enfermera egresada de la Universidad Nacional de Colombia. Especialista en Auditoría en Salud. Magistrada Tribunal Nacional Ético de
Enfermería de Colombia período 2006-2010. Directora de postgrados, Facultad de Enfermería de la Fundación Universitaria de Ciencias de la Salud Bogotá D.C. Colombia. Candidata al doctorado en bioética Universidad El Bosque. 
a culture of self-regulation with the objective of achieving continuous improvement among the academic community. That which several decades ago was a "wearing-out trial", has become a life-style within universities, thus, observing, evaluating, transforming, improving and transcending is a part of growth and development of the communities of higher education institutions. This at least has been the Health Sciences University Foundation perspective. This paper shows the pathway the School of Nursing has crossed in the social, political, and economic disciplinary development of Colombian healthcare through the trace spawned by the research projects of students who attended both clinical and inter-disciplinary specialization programs. We consider it important to present the essence of these steps and pathways to the academic community, considering the Foundation's School of Nursing is ranked upon the country's top twelve supported on three major processes: first, accreditation by the National Ministry of Education; second, being ranked among the top ten resulting from the scores obtained by our students in the ECAESs, since this evaluation system was created five years ago; and third, the positioning and recognition conferred by the employers of public and private entities, who praise our transparency, human, technical and scientific professional quality providing a disciplinary and institutional guarantee validation. Thesis projects presented by graduates from 1997 to the first half of 2008, considering their title, topic, area of interest and methodology, were reviewed.

Key words: trends, research.

\section{Tendencias}

En la actualidad este concepto es importante para el análisis de procesos y productos que tienen que ver con el mercado tanto de bienes como de servicios. Significa "un patrón de comportamiento de los elementos de un entorno particular durante un periodo de tiempo", otras definiciones encontradas y que tuvieron un sentido particular, son: "término que hace referencia a evolución", "fuerza que impulsa a una persona o cosa hacia un fin o lugar".

Bajo esa perspectiva la investigación debe estar presente en la formación de los profesionales, puesto que debe desarrollarse en el estudiante y futuro profesional la capacidad de asombro, la pregunta constante, la inquietud por confirmar o negar tanto la teoría como la práctica para motivar la lectura permanente y es aquí donde cada facultad o programa debe propender por un objetivo, un sendero, un camino y es a esa ruta que se le denomina tendencia educativa o formativa.

Investigar supone aplicar la inteligencia a la exacta comprensión de la realidad objetiva con el fin de dominarla. Sólo al captar la esencia de las cosas, al confrontarla con la realidad, se cumple la labor del investigador. La consecuencia de tal proceso incrementará los conocimientos científicos que dan claridad, identidad y competencia al profesional. Teniendo en cuenta que la profesión de enfermería tiene un enfoque eminentemente so- cial, requiere incrementar los conocimientos teóricos, metodológicos y técnicos que le den validez a su práctica social. Para definir su marco teórico, es indudable la necesidad de aplicar la metodología de investigación como parte del deber ser académico, práctico y asistencial. Se ha mencionado a través de la revisión de literatura que la investigación en enfermería constituye un campo de enormes posibilidades de desarrollo, por lo cual es necesario introducir al estudiante y al profesional en el estudio del método científico, con el fin de lograr tal apropiación de los conocimientos y sensibilidad investigativa que lo aplique de manera permanente en sus labores cotidianas, tanto docentes como asistenciales y administrativas.

Rojas Soriano afirma "la investigación es una búsqueda ordenada de conocimientos, coherente, de reflexión analítica y confrontación continua de los datos empíricos y el pensamiento abstracto, a fin de explicar los fenómenos de la naturaleza". El mismo autor explica "para descubrir las relaciones e interconexiones básicas a que están sujetos los procesos y los objetos, es necesario el pensamiento abstracto, cuyo producto (conceptos, hipótesis, leyes, teorías) debe ser sancionado por la experiencia y la realidad concreta...”. Por esta razón, para la educación y en particular la de profesionales prestadores de servicios en salud como son nuestras enfermeras, es importante entender, conocer y marcar "la tendencia de formación" de acuerdo no sólo con las necesidades socio-laborales, sino también en correspondencia con las 
necesidades de desarrollo humano y social más allá de lo que se tiene en el presente, para que nuestros egresados promuevan servicios, programas, proyectos y políticas transformadoras, y no para que ejerzan sólo como "enfermeras...bueno... si...bueno" obedecedoras de prescripciones médicas. No queriendo decir que en la dialógica intersubjetiva del equipo de salud no exista la necesidad de ejercer acciones dependientes, interdependientes e independientes, autónomas, fundamentadas en el conocimiento con alto sentido ético de compromiso, responsabilidad, solidaridad y respeto.

\section{La investigación en el conocimiento y la práctica de enfermería}

Donaldson y Bottorff expresan frente a la investigación en enfermería que "es la fuente de desarrollo de conocimiento que le da la característica de disciplina, que ha de estar presente tanto en la práctica clínica como en el desarrollo de la administración y la educación, que ha de dar respuesta a las necesidades sociales que están relacionadas con procesos de salud-enfermedad, condiciones y calidad de vida de las personas, familias y comunidades, en todos los grupos etarios. En la actualidad emergen para el siglo XXI tres temas generales para investigar, en los cuales debe interesarse el profesional de enfermería y los estudiantes: 1) en los principios y leyes que gobiernan los procesos de la vida, bienestar y funcionamiento óptimo de los seres humanos enfermos o sanos; 2) en los patrones de comportamiento humano y su interacción con el medio ambiente en situaciones críticas de la vida, y 3 ) en los procesos por los cuales se afectan los campos positivos del estado de salud.

Sin embargo, según lo propuesto por Donaldson y Bottorff en sus artículos lo que se encuentra en la producción investigativa de Colombia, Latinoamérica y el mundo, hay todavía una gran distancia frente a las líneas propuestas y los trabajos desarrollados. Según el CIE, las prioridades de investigación en enfermería para el presente siglo son: 1) generar conocimiento disciplinar, el cual se relaciona con el desarrollo y aplicación de modelos conceptuales y teorías de enfermería; 2) desarrollar estrategias para mejorar los programas de promoción de la salud y prevención de la enfermedad, en especial lo que corresponde a la determinación de factores de riesgo, incluyendo la vigilancia y control de los mismos; 3) establecer características de las poblaciones y evaluar estrategias encaminadas a la modificación de factores de riesgo, puesto que se han dinamizado y transformado en los últimos años; 4) analizar aspectos relacionados con la prestación de servicios de salud, dentro de lo cual se sugiere mejorar la calidad del cuidado de enfermería y estudiar el impacto de las intervenciones de enfermería en las metas de salud y bienestar; 5) analizar la calidad de vida laboral de las enfermeras, la retención, movilidad local, regional e internacional, satisfacción laboral, estrés laboral, relación de enfermeras con la población, etc.; 6) participar en el desarrollo de políticas encaminadas a la equitativa, oportuna y eficiente prestación de servicios de salud, y 7) integrar la práctica basada en la evidencia, lo cual dinamiza y amplía los horizontes de cuidado, puesto que surge desde escenarios distintos y promueve el trabajo en equipo entre la docencia y la asistencia.

Alrededor de estos planteamientos existe una mayor sincronía en torno a los trabajos desarrollados por las enfermeras en la producción nacional e internacional. No obstante, en la revisión de la literatura se encuentra que la producción investigativa tiene que ver con las temáticas consignadas en las Tablas 1 y 2 .

Dentro de la información que nos aporta cada uno de los documentos se puede observar que hay diferencia en la prioridad de la investigación. Tenemos por ejemplo, que el estudio mundial se centra en la investigación referente al cuidado/práctica de enfermería encontrando 88 trabajos $(77,8 \%)$ y que por grupos de edad el cuidado de enfermería al adulto es el más investigado con 71 publicaciones $(62,5 \%)$. Se puede ver que en enfermería es mucho lo que hay para investigar en torno a los temas abordados por las políticas disciplinares nacionales e internacionales y de prestación de servicios de salud, tanto desde la formación como la práctica. Por ejemplo, frente a las metas del milenio establecidas por el CIE y por entidades como la Organización Mundial de la Salud(OMS), el Banco Mundial (BM), la Organización Panamericana de la Salud (OPS), el Ministerio de la Protección Social de Colombia, no se están asumiendo los procesos de investigación que permitan en los próximos años dar res- 


\section{Tabla I. Clasificación temática de las investigaciones en el mundo, 1988-1992}

\begin{tabular}{|c|c|c|c|c|c|c|}
\hline Características & 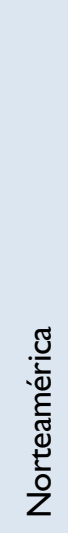 & 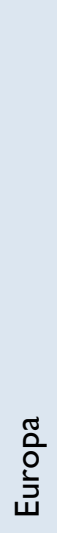 & 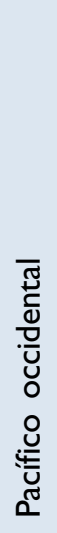 & 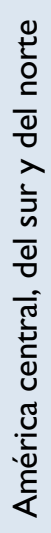 & 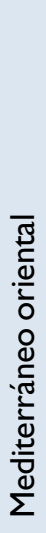 & סु \\
\hline Cuidado de enfermería & 48 & 21 & 4 & 9 & 6 & 88 \\
\hline Práctica de enfermería & 46 & 20 & 3 & 8 & 5 & 82 \\
\hline Teoría de enfermería & 34 & 10 & 4 & 6 & 5 & 59 \\
\hline Enfermería & 36 & 10 & 4 & 7 & 6 & 63 \\
\hline Dirección organizacional y administración & 21 & 6 & 4 & 5 & 4 & 40 \\
\hline Educación en enfermería & 31 & 12 & 3 & 6 & 7 & 59 \\
\hline Metodología de la investigación & 37 & 13 & 3 & 6 & 5 & 64 \\
\hline
\end{tabular}

Tabla 2. Clasificación temática de las investigaciones presentadas en los Coloquios Panamericanos, 1988-1998

\begin{tabular}{|l|c|c|}
\hline Tema & No. & $\%$ \\
\hline Salud pública & 84 & 32 \\
\hline Recursos humanos & 62 & 23,6 \\
\hline Clínica & 36 & 13,7 \\
\hline Administración & 29 & 11,9 \\
\hline Cuidado de enfermería & 16 & 6,1 \\
\hline Producción científica & 15 & 5,7 \\
\hline Estudios interpretativos & 12 & 4,6 \\
\hline Aspectos éticos legales & 6 & 2,3 \\
\hline Historia & 3 & 1,1 \\
\hline
\end{tabular}

puesta a las necesidades de prestación de servicios en eficiencia, oportunidad y calidad.

En cuanto a la investigación generada en las especializaciones de la Facultad de Enfermería de la Fundación Universitaria de Ciencias de la Salud desarrolladas entre 1997 y 2008 se elaboraron 103 trabajos, de los cuales el
$99 \%$ abordan el adulto como sujeto de cuidado, por lo cual es necesario explicar que la orientación de las especializaciones clínicas en la facultad es el cuidado del adulto, pero cuando existe interés por parte de alguno de los grupos de investigadores para desarrollar trabajos con niños, éstos se han respaldado con el equipo de docentes del área pediátrica de la facultad, situación que se ha 
presentado en cuatro ocasiones. Teniendo en cuenta nuestra filosofía y perfil institucional, el $89 \%$ de los trabajos realizados tienen que ver con el quehacer y práctica de enfermería desde la visión clínica, el porcentaje restante corresponde a la producción de la especialización de gerencia de la salud la cual es de carácter interdisciplinar. También se evidencia que cada vez más los estudiantes se motivan por desarrollar investigación en temas como prácticas de salud, aspectos de educación en enfermería, al paciente en estado con enfermedades crónicas y con menor frecuencia temas como gestión, gerencia y administración del cuidado, así como la aplicación, uso y significado de los modelos y teorías de enfermería.

\section{Diseños metodológicos de las investigaciones}

En el informe de las investigaciones a nivel mundial se encuentran los descritos en la Tabla 3. En cuanto a los coloquios, no es posible evidenciar los diseños metodológicos, aunque en el resumen sobre los trabajos las autoras expresan que son estudios monodisciplinares con enfoque cuantitativo, descriptivos, de colectivos de investigadores sin adscripción a líneas o programas de investigación.

La metodología más frecuente en el escenario de la FUCS corresponde a la investigación cuantitativa que es compatible con el modelo de enseñanza y con la habilidad investigativa de los docentes por su perfil clínico. Sin embargo, cada día aumentan las metodologías cualitativas sobre todo del tipo descriptivo. Así mismo se evidencia en la producción investigativa de la FUCS que los estudios son monodisciplinares, lo cual se encuentra dentro del marco de la producción latinoamericana de la cual debemos trascender.

En cuanto al nivel de producción, la información aportada en los diferentes documentos demuestra que la mayor trayectoria y productividad académica-investigativa se encuentra en los países desarrollados. Sin embargo,

\begin{tabular}{|c|c|c|c|c|c|c|}
\hline Características & 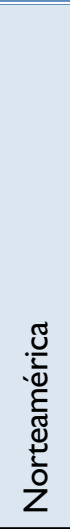 & 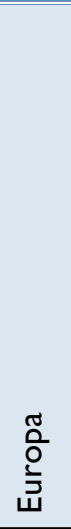 & 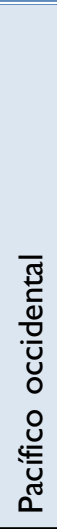 & 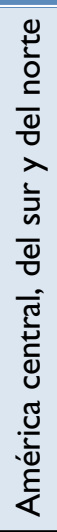 & 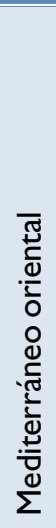 & 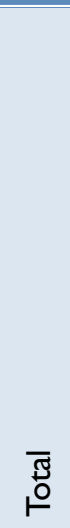 \\
\hline No. total de unidades de investigación & 61 & 27 & 6 & 12 & 8 & 114 \\
\hline Encuestas & 44 & 18 & 5 & 7 & 7 & 81 \\
\hline Histórica/ética & 14 & 6 & 3 & 4 & 3 & 30 \\
\hline Experimental/cuasi-experimental & 55 & 16 & 2 & 4 & 5 & 82 \\
\hline Evaluaciones/necesidades de valoración & 35 & 12 & 3 & 6 & 6 & 62 \\
\hline Correlacional & 33 & 7 & 2 & 4 & 4 & 50 \\
\hline Epidemiológica & 12 & 7 & $\mathrm{I}$ & 2 & 5 & 27 \\
\hline Cualitativa fenomenológica & 33 & 14 & 3 & 3 & 2 & 55 \\
\hline Aplicación de sistemas & 9 & 4 & - & 3 & 2 & 18 \\
\hline Otras & 9 & - & - & - & - & 9 \\
\hline
\end{tabular}


Latinoamérica ha generado en muy corto tiempo una alta participación, a la que estamos llamados a contribuir los docentes y enfermeros de la FUCS.

Es necesario también tener en cuenta que esta trayectoria y productividad evidencia la necesidad de crecimiento y desarrollo desde la academia y la asistencia en la formación conceptual y metodológica investigativa, lo cual se ve reflejado en el número de programas y profesionales formados en especializaciones que afinan el conocimiento y la técnica disciplinar, las maestrías y los doctorados que intervienen en la formación, la práctica y las necesidades sociales.

\section{Conclusiones}

Para lograr que la investigación en la Fundación Universitaria de Ciencias de la Salud (FUCS), en Colombia y en Latinoamérica sea una realidad constante, progresiva, trascendente y transformadora es necesario desde las aulas de las universidades generar estrategias fundamentales que se encuentran descritas en forma implícita y explícita en la literatura revisada, en donde encontramos estrategias como:

- Enseñar la enfermería como ciencia mediante metodologías como aprendizaje significativo, analítico, argumentativo, crítico, propositivo, de solución de problemas, entre otros, para lo cual es trascendente enseñar a leer, escribir y pensar a nuestros estudiantes y enfermeras.

- Desarrollar competencias investigativas según el nivel de formación de los estudiantes integrando ejercicios teórico-prácticos, lo cual desarrollado de manera dinámica permitirá que en el profesional de enfermería haya conciencia y motivación investigativa.

- Incentivar la lectura, asimilación y apropiación de la producción investigativa no sólo entre los profesionales de enfermería o de la salud, sino entre la sociedad en general, para lo cual es necesario dinamizar el estilo de presentación de los informes y la calidad del lenguaje, lo cual no ha de disminuir el rigor científico.
- Promover o desarrollar las unidades, centros y/o redes de investigación entre las docentes y las enfermeras de asistencia, lo que contribuirá al desarrollo de la investigación disciplinar y colaborativa, así como propender por el desarrollo de la investigación inter y transdisciplinar.

- Generar mayores espacios de publicación para la producción académica e investigativa de las enfermeras y favorecer que esos espacios de publicación sean asequibles tanto al personal académico, como de interés y entendimiento para la comunidad en general.

- Promover el mejoramiento de la práctica disciplinar, teniendo como evidencia-referencia la producción investigativa.

- Promover entre los docentes, estudiantes y enfermeras de la asistencia la utilización de los medios tecnológicos de información, para dinamizar el flujo y consecución de la producción académica e investigativa.

- Participar en forma dinámica en la definición de políticas de salud, desarrollo, educación e inversión social, a nivel local, regional, nacional e internacional.

- Promover y participar en los eventos académicos que permiten la difusión de los productos tanto académicos como investigativos.

Para finalizar, el patrón o fuerza productiva académica e investigativa de nuestra Fundación para la enfermería colombiana, latinoamericana y mundial debe estar enmarcada en la resolución de problemáticas sociales (salud, educación, nutrición, cuidado y preservación del medio ambiente), la generación e intervención en políticas públicas nacionales y mundiales acordes con las necesidades reales y potenciales de la población, la renovación del conocimiento disciplinar, el empoderamiento profesional, la mejoría de la imagen, idoneidad y dignidad profesional, el desarrollo de modelos y/o teorías locales, y la construcción de una mejor calidad de vida para nuestros profesionales y usuarios de los servicios de salud, desde el poder que da en cada uno de nosotros ejercer nuestras funciones labo- 
rales con idoneidad, eficiencia, motivación, compromiso, responsabilidad y calidad desde donde nos encontremos, ya sea en la docencia o en la asistencia.

\section{Lecturas recomendadas}

- Benner P. From novice to expert: excellence and power in clinical nursing practice. California: Addison- Wesley Publishing Company; 1984.

- Bottorff JL. Nursing: a practical science of caring. ANS Adv Nurs Sci. 1991 Sep;14(1):26-39.

- Burns N, Grove S. Investigación en enfermería. 3a ed. Barcelona: Elsevier; 2004.

- Castrillón C, Nájera RM, Orrego Sierra S. Estado del arte de la investigación presentada en los Coloquios Panamericanos de Investigación en enfermería. En: Memorias del 7 Coloquio Panamericano de Investigación en Enfermería; 2000 Oct. 9-12; Bogotá: ACOFAEN; 2000. pp. 86-94.

- Consejo Internacional de Enfermería. La investigación en enfermería: instrumento de acción [monografía en Internet]. Ginebra: CIE; 200? [ [citado 29 abr. 2009]. Disponible en: http://www.icn.ch/matters_researchsp.htm
- Donaldson SK, Crowley DM. The discipline of nursing. Nurs Outlook. 1978 Feb;26(2):113-20.

- Duran de Villalobos MM. Enfermería: desarrollo teórico e investigativo. Bogotá: Universidad Nacional de Colombia. Facultad de Enfermería; 1998.

- Hernández de Canales F, Pineda EB. Metodología de la investigación, manual para el desarrollo de personal de salud. México: Limusa; 2005

- Jiménez MA, Gómez Serrano C. Contexto de la producción investigativa en educación en enfermería en Colombia 1995 - 2004. Av. Enferm. 2005 Ene-Jun; 23(1): 5-17.

- Johnson JL. Nursing science: basic, applied, or practical?: implications for the art of nursing. ANS Adv Nurs Sci. 1991 Sep; 14(1):7-16.

- Kim MI. Nursing research: a worldwide picture. Rev Esc Enferm USP. 1992 Oct;26(Suppl):7-22.

- Meleis AI. Theorical nursing: development and progress. 4th ed. Philadelphia: Lippincott Williams \& Wilkins; 2006.

- Sánchez Herrera B, Villaraga L, et al. La investigación y el cuidado en América Latina. Bogotá: Universidad Nacional de Colombia. Facultad de Enfermería; 2005. 